\title{
The Clinical Features and Emotional Stressors in Korean Patients with Tako-Tsubo Cardiomyopathy
}

\author{
Bong Gun Song, Ju Hyeon Oh, Yong Hwan Park, Gu Hyun Kang, and Woo Jung Chun \\ Division of Cardiology, Cardiac and Vascular Center, Department of Medicine, Samsung Changwon Hospital and \\ Sungkyunkwan University School of Medicine, Changwon 630-522, Republic of Korea \\ Correspondence should be addressed to Ju Hyeon Oh, ojh@korea.com
}

Received 11 July 2012; Revised 6 August 2012; Accepted 7 August 2012

Academic Editor: Richard C. Veith

Copyright ( $(2012$ Bong Gun Song et al. This is an open access article distributed under the Creative Commons Attribution License, which permits unrestricted use, distribution, and reproduction in any medium, provided the original work is properly cited.

\begin{abstract}
Background. Tako-tsubo cardiomyopathy (TTC) is typically triggered by an acute emotional or physical stress events. Aim of this study was to investigate the impact of emotional stressors on clinical features, laboratory parameters, electrocardiographic and echocardiographic findings in patients with TTC. Methods. Of 103 patients enrolled from the TTC registry database, fifteen patients had emotional triggers (E group), and 88 patients had physical triggers or no triggers (other group). Results. Most clinical presentations and in-hospital courses were similar between the groups. However, E group had higher prevalence of chest pain $(87$ versus $42 \%, P=0.001$ ), palpitation (27 versus $6 \%, P=0.008$ ), whereas other group had higher prevalence of cardiogenic shock (35 versus $7 \%, P=0.027$ ). E group had significantly higher corrected QT intervals (median, 477.5 versus $438 \mathrm{~ms}, P=0.001$ ), and left ventricular ejection fraction (LVEF) (mean, 45.7 versus $39.6 \%, P=0.001$ ), but lower hs-CRP (median, 0.1 versus $3.3 \mathrm{mg} / \mathrm{L}$, $P=0.001$ ), CK-MB (median, 5.5 versus $11.9 \mathrm{ng} / \mathrm{mL}, P=0.047$ ), troponin-I (median, 1.0 versus $3.2 \mathrm{ng} / \mathrm{mL}, P=0.011$ ), and NT-proBNP levels (median, 2145 versus $4939 \mathrm{pg} / \mathrm{mL}, P=0.020$ ). Other group required more frequent hemodynamic support and had significantly longer intensive care unit (median, 3 versus 1 days, $P=0.005$ ) and in-hospital (median, 17 versus 3 days, $P=0.001$ ) durations. Conclusion. The clinical features of TTC are different between groups with and without preceding emotional stressors. The TTC group with preceding emotional stressors was more likely to have preserved cardiovascular reserve and lesser likely to require hemodynamic support than other group although the entire prognosis of TTC is excellent regardless of triggering stressors.
\end{abstract}

\section{Introduction}

Tako-tsubo cardiomyopathy (TTC), also known as transient left ventricular (LV) ballooning syndrome, or stress-induced cardiomyopathy, is characterized by transient LV dysfunction in the absence of significant angiographic coronary stenoses, typically triggered by preceding emotional or physical stress [1-4]. It has been typically observed in postmenopausal females experiencing an emotionally stressful event inspiring to name this condition also broken heart syndrome and is increasingly being observed under diverse circumstances, including medical/surgical procedures or diagnostic test [57]. Differences in these clinical presentations have been observed, suggesting the possibility of diverse clinical phenotypes according to triggering stressors [6-8]. However, there have been few data to review and analyze the similarities and differences of clinical features between patients with TTC presenting with and those without preceding emotional stressors [9]. In this study, we investigated the impact of emotional stressors on clinical characteristics, laboratory parameters, electrocardiographic and echocardiographic findings in patients with TTC.

\section{Materials and Methods}

2.1. Study Subjects. We approached 103 consecutive patients enrolled from the TTC registry database at Samsung Changwon Hospital from January 2004 to December 2009. From 5078 consecutive patients with a diagnosis of an acute coronary syndrome, including ST- and non-ST-elevation myocardial infarction, who had an urgent coronary angiography (CAG), 103 (2\%) patients were diagnosed with TTC. 
The enrolled 103 patients with TTC were divided into the following two subgroups according to the presence or absence of emotional stressors: fifteen (15\%) patients had emotional triggers (E group), 88 patients had physical triggers or no triggers (other group). The criteria for inclusion were as follows: (1) transient akinesia/dyskinesia beyond a single major coronary artery vascular distribution, (2) absence of significant coronary artery disease on coronary angiograms (diameter stenosis $<50 \%$ by visual estimation) or angiographic evidence of acute plaque rupture, and (3) new electrocardiographic changes (ECG) (ST-segment changes, T-wave inversion, or Q-wave) [10]. Cardiogenic shock was defined as a systolic blood pressure $<90 \mathrm{mmHg}$ for $\geq 30$ minutes that was not responsive to fluid administration alone, accompanied by evidence of tissue hypoperfusion in the setting of clinically adequate or elevated LV filling pressures [11]. Pulmonary edema was defined as the presence of rales at pulmonary examination or a radiographic report of pulmonary alveolar/interstitial congestion at initial chest roentgenogram [11]. Hypertension was defined as repeated measurements of $\geq 140 \mathrm{mmHg}$ SBP or $\geq 90 \mathrm{mmHg}$ DBP, or previous antihypertensive drug treatment. Diabetes mellitus was defined as serum glucose level of $125 \mathrm{mg} / \mathrm{dL}$ or higher, a history of diabetes mellitus, or current use of antidiabetic therapy. Current smoking was defined as having smoked cigarettes less than 1 year before patients presented with TTC. The protocol was approved by the Institutional Research Ethics Committee. The recommendations of the revised version of the Declaration of Helsinki were met.

2.2. Methods. The medical history and coronary risk factors were obtained from medical records combined with a patient questionnaire. Any physical or emotional stresses prior to the onset of this syndrome were specifically investigated. ECG and laboratory data including cardiac enzymes (creatine kinase $[\mathrm{CK}]$, creatine kinase $\mathrm{MB}$ fraction $[\mathrm{CK}-\mathrm{MB}]$, and troponin-I) were recorded during the acute phase and were followed until the abnormalities disappeared. Followup data were collected by direct telephone interviews and a detailed review of all medical records. The cause and data of death were confirmed by information from the National Population Registry of the Korea National Statistical Office, together with a review of all available clinical records at the time of death.

2.3. Echocardiography. Transthoracic echocardiographic (TEE) examinations were performed in all patients with a $2.5 \mathrm{MHz}$ transducer attached to a commercially available Doppler echocardiography machine, on the first hospital day or within 24 hours of CAG and followup. LV end diastolic (LVEDD) and end systolic diameters (LVESD), along with septal and posterior wall thickness at end diastole were measured in the parasternal long axis view, using 2D-guided M-mode echocardiography according to the recommendations of chamber quantification [12]. The LV ejection fraction (EF) was calculated by modified Simpson's method. The valvular regurgitation (VR) was assessed by color Doppler flow mapping of spatial distribution of the regurgitant jet in accordance with the ASE recommendation [13]. In our study, significant VR was defined as regurgitation of more than a mild degree. Reversible VR was defined as significant VR at initial echocardiogram that appeared at followup echocardiogram. Right ventricular systolic pressure (RVSP) was derived from tricuspid regurgitation gradient measured in accordance with the ASE recommendation [13]. In our study, we assessed the presence of systolic anterior motion (SAM) of the mitral valve using 2-dimensional imaging [14]. Left atrial (LA) volume was determined by the prolate ellipse method and indexed by body surface area (LAVI).

\subsection{N-Terminal Probrain Natriuretic Peptide (NT-proBNP) Assay. We took blood samples from the antecubital vein using lithium heparin, and the blood samples were then centrifuged. The blood samples were stored at $-70^{\circ} \mathrm{C}$ until further analysis. Plasma NT-proBNP levels were measured using an Elecsys pro BNP reagent kit (Roche Diagnostics, USA) and an Elecsys 2010 (Roche Dignostics, USA). In all cases, the time interval between blood sampling for NT- proBNP and echocardiography was within 1 day.}

2.5. Statistical Analysis. Statistical analyses were performed using SPSS statistical software (version 11.0, SPSS Corp, Chicago, IL, USA). Quantitative data are presented as mean \pm standard deviation. Age, heart rate, corrected QT interval, hs-CRP levels, peak CK levels, peak CK-MB levels, peak troponin-I levels, left ventricular end diastolic pressure (LVEDP), duration of hospitalization, and duration of ICU stay are given in terms of the median and interquartile range (IQR). Qualitative data are presented as frequencies. The student's $t$-test or the Mann-Whitney test was used to compare the continuous variables and the chi-square test was used to compare the categorical variables. All $P$ values are two tailed and differences were considered significant when the $P$ value was less than 0.05 .

\section{Results}

3.1. The Comparison of Clinical Characteristics between the Subgroups. Emotional stressors were documented in $15 \%$ of patients $(15 / 103)$, whereas physical stressors were documented in $67 \%$ of patients $(69 / 103)$ and $18 \%$ of patients $(19 / 103)$ had no triggering stressors (Figure 1). Among physical stressors, acute medical illness, surgery/procedure, and intravenous drug use were the precipitants in $34 \%$ of patients (35/103), 30\% of patients (31/103), and $3 \%$ of patients (3/103), respectively (Figure 1 ).

The clinical characteristics and initial presentations of the subgroups are compared in Table 1 . The E group had significantly higher prevalence of chest pain (87 versus $42 \%, P=$ 0.001 ), and palpitation (27 versus $6 \%, P=0.008$ ), whereas other group had higher prevalence of cardiogenic shock (35 versus 7\%, $P=0.027$ ) than $\mathrm{E}$ group. The subgroups did not differ significantly in terms of age, male gender, body surface area, the prevalence of underlying diseases such as stroke, liver cirrhosis, hypertension, diabetes mellitus, 


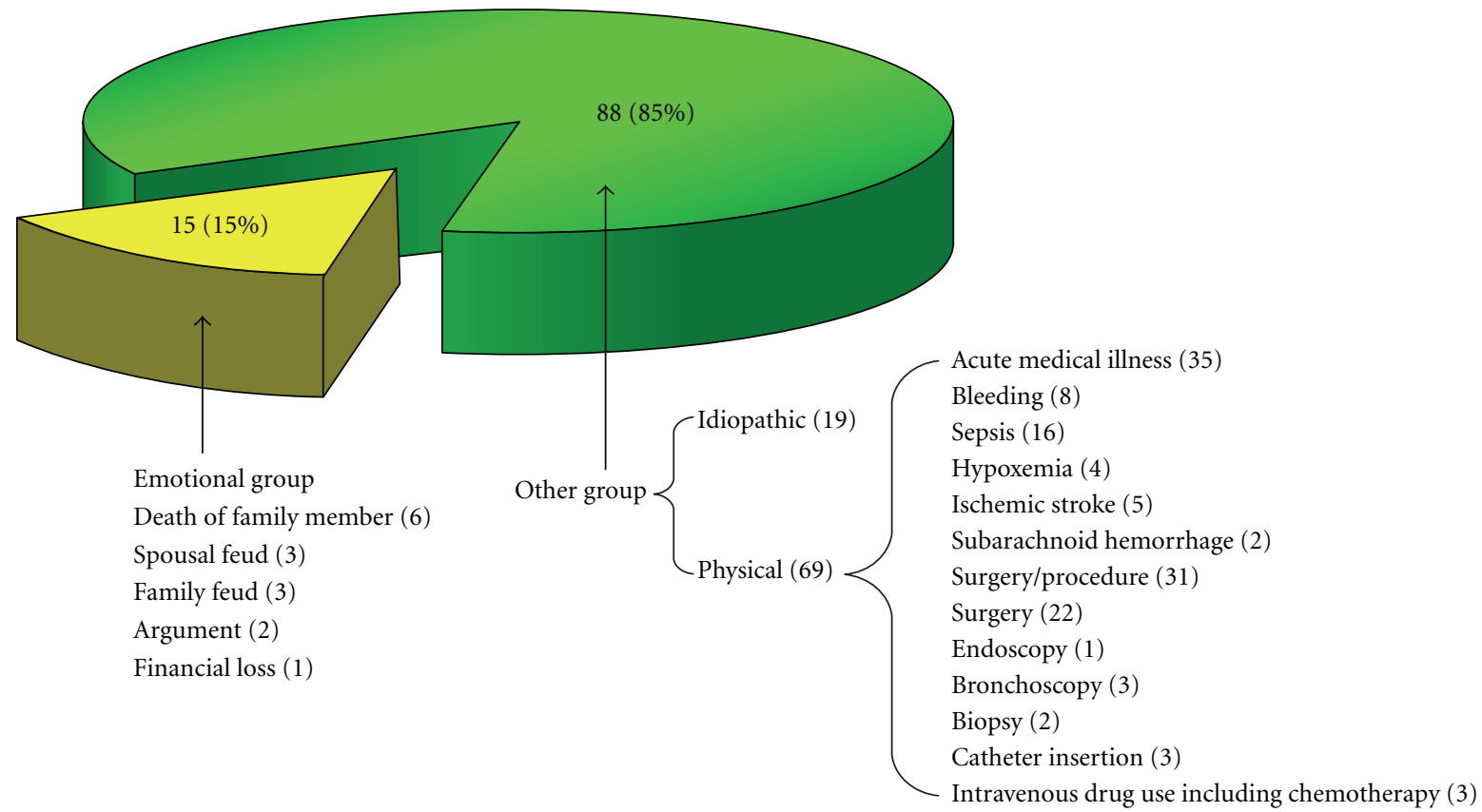

Figure 1: Preceding stressful events of 103 patients with TTC. Emotional stressors were documented in 15\% of patients, whereas physical stressors were documented in $67 \%$ of patients and $18 \%$ of patients had no triggering stressor.

and current smoking status. Also, there were no significant differences in clinical presentations such as dyspnea, loss of consciousness, nausea/vomiting, and pulmonary edema between two groups.

3.2. The Comparison of ECG Changes, Laboratory, and Angiographic Findings between the Subgroups. The ECG changes, laboratory, and angiographic findings of subgroups are compared in Table 2. The E group had significantly higher corrected QT interval (median, 477.5 versus $438.0 \mathrm{~ms}$, $P=0.002$ ), but lower heart rate (median, 84 versus 66 beats per minute, $P=0.001$ ) than other group. The $\mathrm{E}$ group had significantly lower hs-CRP (median, 0.1 versus $3.3 \mathrm{mg} / \mathrm{L}, P=0.001$ ), peak CK-MB (median, 5.5 versus $11.9 \mathrm{ng} / \mathrm{mL}, P=0.047$ ), troponin-I (median, 1.0 versus $3.2 \mathrm{ng} / \mathrm{mL}, P=0.011$ ), NT-proBNP levels (median, 2145.0 versus $4939.0 \mathrm{pg} / \mathrm{mL}, P=0.020$ ), and LVEDP (median, 14 versus $19 \mathrm{mmHg}, P=0.039$ ) than other group. However, there were no significant differences in ECG findings with regard to rhythm abnormalities such as atrial fibrillation, life-threatening arrhythmias, ST segment elevation, T-wave inversion, and Q-wave between two groups.

3.3. The Comparison of Echocardiographic Findings between the Subgroups. The Echocardiographic findings of subgroups are compared in Table 3 . The groups did not differ significantly in terms of ballooning patterns. The E group had significantly higher ejection fraction (mean, 45.7 versus $39.6 \%, P=0.001$ ), and lower LVEDD (mean, 49.5 versus $52.2 \mathrm{pg} / \mathrm{mL}, P=0.009$ ), and LVESD (mean, 37.9 versus $30.8 \%, P=0.001)$ than other group. There were no significant differences in the LAVI, RVSP, and $\mathrm{E} / \mathrm{E}^{\prime}$ on the initial and followup examinations between two groups. Also, there were no significant differences in SAM, significant mitral regurgitation (MR), aortic regurgitation (AR), and tricuspid regurgitation (TR) between two groups on the initial and followup examinations. All patients showed normalized regional wall motion in their followup echocardiogram.

\subsection{The Comparison of Management and Clinical Outcomes} between the Subgroups. There were no significant differences in use of inotropics, use of intra-aortic balloon pump (IABP), use of angiotensin-converting enzyme inhibitor (ACEI) or angiotensin receptor blocker (ARB), and use of beta blocker during hospitalization between the two groups. The other group had significantly higher prevalence of diuretic use, and frequency of ICU stay and had significantly longer durations of in-hospitalization and ICU stay than the E group (Table 4).

During followup (median, 5.1 years, IQR, 4.0-6.1 years), $14(14 \%)$ patients died; 8 patients died of malignancy, 2 of stroke, 2 of chronic renal failure with panperitonitis, 1 of liver cirrhosis with variceal bleeding, and 1 of pneumonia with empyema. However, cardiac deaths associated with LV ballooning syndrome itself were not noted in three groups. Also, recurrence of the LV ballooning syndrome was not noted in two groups.

\section{Discussion}

TTC usually precipitated by an acute episode of emotional and/or physiological stress has been widely reported in the past [1-4]. Recently, diverse clinical features of TTC have been observed, suggesting the possibility of more than one 
TABLE 1: The comparison of clinical characteristics between the subgroups.

\begin{tabular}{|c|c|c|c|c|}
\hline & Total $(n=103)$ & E group $(n=15)$ & Other group $(n=88)$ & $P$ \\
\hline Age $(\text { years })^{\dagger}$ & $59.0(52.0-72.0)$ & $58.0(54.0-78.0)$ & $59.0(52.0-72.0)$ & 0.578 \\
\hline Male gender, $n(\%)$ & $33(32)$ & $2(13)$ & $31(35)$ & 0.093 \\
\hline Body surface area $\left(\mathrm{m}^{2}\right)$ & $1.6 \pm 0.2$ & $1.5 \pm 0.1$ & $1.6 \pm 0.2$ & 0.093 \\
\hline Hypertension, $n(\%)$ & $32(31)$ & $2(13)$ & $30(34)$ & 0.108 \\
\hline Diabetes mellitus, $n(\%)$ & $22(21)$ & $2(13)$ & $20(23)$ & 0.412 \\
\hline Current smoker, $n(\%)$ & $8(8)$ & $2(13)$ & $6(7)$ & 0.384 \\
\hline \multicolumn{5}{|l|}{ Underlying diseases } \\
\hline Stroke/transient ischemic attack, $n(\%)$ & $6(6)$ & $0(0)$ & $6(7)$ & 0.297 \\
\hline Liver cirrhosis, $n(\%)$ & $2(2)$ & $0(0)$ & $2(2)$ & 0.555 \\
\hline Chronic renal failure, $n(\%)$ & $7(7)$ & $0(0)$ & $7(8)$ & 0.258 \\
\hline Malignancy, $n(\%)$ & $18(17)$ & $0(0)$ & $18(20)$ & 0.054 \\
\hline \multicolumn{5}{|l|}{ Clinical presentation } \\
\hline Chest pain, $n(\%)$ & $50(49)$ & $13(87)$ & $37(42)$ & $0.001^{*}$ \\
\hline Dyspnea, $n(\%)$ & $54(52)$ & $9(60)$ & $45(51)$ & 0.525 \\
\hline Palpitation, $n(\%)$ & $9(9)$ & $4(27)$ & $5(6)$ & $0.008^{*}$ \\
\hline Loss of consciousness, $n(\%)$ & $1(1)$ & $0(0)$ & $1(1)$ & 0.678 \\
\hline Nausea/vomiting, $n(\%)$ & $10(10)$ & $0(0)$ & $10(11)$ & 0.169 \\
\hline Cardiogenic shock, $n(\%)$ & $32(31)$ & $1(7)$ & $31(35)$ & $0.027^{*}$ \\
\hline Pulmonary edema, $n(\%)$ & $42(41)$ & $6(40)$ & $36(41)$ & 0.947 \\
\hline
\end{tabular}

${ }^{\dagger}$ Presented as median (interquartile range). ${ }^{*}$ Significant finding.

TABLE 2: The comparison of electrocardiographic changes, laboratory, and angiographic findings between the subgroups.

\begin{tabular}{|c|c|c|c|c|}
\hline & Total $(n=103)$ & E group $(n=15)$ & Other group $(n=88)$ & $P$ \\
\hline \multicolumn{5}{|l|}{ Electrocardiographic changes } \\
\hline Life-threatening arrhythmia, $n(\%)$ & $16(16)$ & $0(0)$ & $16(18)$ & 0.072 \\
\hline Heart rate $(\text { beats/min })^{\dagger}$ & $80.0(66.0-91.0)$ & $66.0(58.0-72.0)$ & $84.0(71.0-95.0)$ & $0.001^{*}$ \\
\hline Atrial fibrillation, $n(\%)$ & $6(6)$ & $0(0)$ & $6(7)$ & 0.297 \\
\hline Corrected QT interval $(\mathrm{ms})^{\dagger}$ & $438.0(422.8-486.0)$ & $477.5(434.0-544.0)$ & $438.0(417.0-482.3)$ & $0.002^{*}$ \\
\hline ST-segment elevation, $n(\%)$ & $73(71)$ & $13(87)$ & $60(68)$ & 0.145 \\
\hline Q-wave, $n(\%)$ & $13(13)$ & $0(0)$ & $13(15)$ & 0.111 \\
\hline T-wave inversion, $n(\%)$ & $79(77)$ & $12(80)$ & $67(76)$ & 0.744 \\
\hline \multicolumn{5}{|l|}{ Laboratory findings } \\
\hline hs-CRP $(\mathrm{mg} / \mathrm{L})^{\dagger}$ & $2.3(0.8-11.1)$ & $0.1(0.1-10.0)$ & $3.3(1.0-11.4)$ & $0.001^{*}$ \\
\hline NT-proBNP $(\mathrm{pg} / \mathrm{mL})^{\dagger}$ & $3210.0(1102.0-30181.0)$ & $2145.0(613.3-5059.0)$ & $4939.0(1458.0-33017.0)$ & $0.020^{*}$ \\
\hline \multicolumn{5}{|l|}{ Cardiac enzymes } \\
\hline Peak CK $(n g / m L)^{\dagger}$ & $279.0(93.0-632.0)$ & $279.0(519.0-619.5)$ & $270.5(90.0-607.0)$ & 0.421 \\
\hline Peak CK-MB $(\mathrm{ng} / \mathrm{mL})^{\dagger}$ & $11.9(2.8-33.9)$ & $5.5(1.5-23.7)$ & $11.9(3.8-34.8)$ & $0.047^{*}$ \\
\hline Peak troponin-I $(\mathrm{ng} / \mathrm{mL})^{\dagger}$ & $2.0(0.2-11.1)$ & $1.0(0.1-3.3)$ & $3.2(0.2-13.3)$ & $0.011^{*}$ \\
\hline \multicolumn{5}{|l|}{ Angiographic findings } \\
\hline LVEDP $(\mathrm{mmHg})^{\dagger}$ & $19.0(13.0-25.0)$ & $14.0(9.0-20.0)$ & $19.0(13.0-25.0)$ & $0.039 *$ \\
\hline
\end{tabular}

Life-threatening arrhythmia: third-degree atrioventricular block, ventricular tachycardia, ventricular fibrillation, cardiac arrest; LVEDP: left ventricular end diastolic pressure.

${ }^{\dagger}$ Presented as median (inter-quartile range). * Significant finding.

clinical phenotypes according to triggering stressors [6-8]. However, a limited amount of literature currently exists on the similarities and differences of clinical features between patients with TTC presenting with and those without a preceding emotional stressors [9].

To the best of our knowledge, this is one of the largest studies investigating the similarities and differences of clinical features, laboratory parameters, electrocardiographic and echocardiographic findings between patients with TTC presenting with and those without emotional stressors. In the present study, E group had significantly higher prevalence of chest pain and palpitation, and higher corrected QT intervals and LVEF, whereas other group had significantly higher prevalence of cardiogenic shock and higher hs-CRP, CK-MB, 
TABLE 3: The comparison of echocardiographic findings between the subgroups.

\begin{tabular}{|c|c|c|c|c|}
\hline & Total $(n=103)$ & E group $(n=15)$ & Other group $(n=88)$ & $P$ \\
\hline \multicolumn{5}{|l|}{ Initial TTE findings } \\
\hline \multicolumn{5}{|l|}{ Ballooning pattern } \\
\hline Typical TTC pattern, $n(\%)$ & $79(77)$ & $11(73)$ & $68(77)$ & 0.554 \\
\hline Inverted TTC pattern, $n(\%)$ & $20(19)$ & $4(27)$ & $16(18)$ & \\
\hline Mid-ventricular pattern, $n(\%)$ & $4(4)$ & $0(0)$ & $4(5)$ & \\
\hline Localized pattern, $n(\%)$ & $0(0)$ & $0(0)$ & $0(0)$ & \\
\hline $\operatorname{LVEF}(\%)$ & $40.5 \pm 8.0$ & $45.7 \pm 4.3$ & $39.6 \pm 8.2$ & $0.001^{*}$ \\
\hline LVEDD (mm) & $51.8 \pm 6.1$ & $49.5 \pm 2.7$ & $52.2 \pm 6.9$ & $0.009^{*}$ \\
\hline LVESD (mm) & $36.9 \pm 7.0$ & $30.8 \pm 4.8$ & $37.9 \pm 6.8$ & $0.001^{*}$ \\
\hline LAVI $\left(\mathrm{mL} / \mathrm{m}^{2}\right)$ & $28.2 \pm 14.7$ & $22.0 \pm 3.8$ & $29.3 \pm 15.6$ & 0.077 \\
\hline RVSP (mmHg) & $33.7 \pm 3.6$ & $33.4 \pm 3.2$ & $33.8 \pm 3.7$ & 0.696 \\
\hline $\mathrm{E} / \mathrm{E}^{\prime}$ & $10.8 \pm 5.5$ & $10.1 \pm 2.9$ & $11.0 \pm 5.8$ & 0.638 \\
\hline SAM & $13(13)$ & $3(20)$ & $10(11)$ & 0.352 \\
\hline Significant MR, $n(\%)$ & $25(24)$ & $5(33)$ & $20(23)$ & 0.376 \\
\hline Significant AR, $n(\%)$ & $6(6)$ & $2(13)$ & $4(5)$ & 0.179 \\
\hline Significant TR, $n(\%)$ & $15(15)$ & $4(27)$ & $11(13)$ & 0.151 \\
\hline \multicolumn{5}{|l|}{ Follow-up TTE findings } \\
\hline $\operatorname{LVEF}(\%)$ & $62.9 \pm 4.7$ & $65.4 \pm 5.1$ & $62.5 \pm 4.5$ & 0.061 \\
\hline LVEDD (mm) & $49.7 \pm 5.3$ & $50.4 \pm 5.0$ & $49.6 \pm 5.4$ & 0.664 \\
\hline LVESD (mm) & $31.2 \pm 4.2$ & $29.6 \pm 4.8$ & $31.0 \pm 4.1$ & 0.187 \\
\hline LAVI $\left(\mathrm{mL} / \mathrm{m}^{2}\right)$ & $27.6 \pm 14.2$ & $25.8 \pm 3.4$ & $27.7 \pm 15.2$ & 0.684 \\
\hline RVSP (mmHg) & $31.8 \pm 4.7$ & $31.7 \pm 4.7$ & $32.0 \pm 4.0$ & 0.280 \\
\hline $\mathrm{E} / \mathrm{E}^{\prime}$ & $10.5 \pm 5.3$ & $11.7 \pm 3.1$ & $9.5 \pm 3.3$ & 0.478 \\
\hline SAM & $0(0)$ & $0(0)$ & $0(0)$ & - \\
\hline Significant MR, $n(\%)$ & $9(9)$ & $2(13)$ & $7(8)$ & 0.495 \\
\hline Reversible MR, $n(\%)$ & $16(16)$ & $3(20)$ & $13(15)$ & 0.605 \\
\hline Significant AR, $n(\%)$ & $4(4)$ & $0(0)$ & $4(5)$ & 0.400 \\
\hline Significant TR, $n(\%)$ & $9(8)$ & $1(7)$ & $8(9)$ & 0.224 \\
\hline
\end{tabular}

TTE: transthoracic echocardiography; LVEF: left ventricular ejection fraction; LVEDD: left ventricular end-diastolic diameter; LVESD: left ventricular endsystolic diameter; LAVI: left atrial volume index; RVSP: right ventricular systolic pressure; E/E': early diastolic mitral inflow velocity/early diastolic annular velocity; SAM: systolic anterior motion of anterior mitral leaflet; MR: mitral regurgitation; TRL: tricuspid regurgitation; AR: aortic regurgitation.

* Significant finding.

TABLE 4: The comparison of clinical courses and management between the subgroups.

\begin{tabular}{|c|c|c|c|c|}
\hline & Total $(n=103)$ & E group $(n=15)$ & Other group $(n=88)$ & $P$ \\
\hline Use of inotropics, $n(\%)$ & $28(27)$ & $2(13)$ & $26(30)$ & 0.192 \\
\hline Use of IABP, $n(\%)$ & $12(12)$ & $0(0)$ & $12(14)$ & 0.128 \\
\hline Use of ACEI or ARB, $n(\%)$ & $57(55)$ & $10(67)$ & $47(53)$ & 0.340 \\
\hline Use of beta blocker, $n(\%)$ & $21(20)$ & $4(27)$ & $17(19)$ & 0.514 \\
\hline Use of diuretic, $n(\%)$ & $40(39)$ & $2(13)$ & $38(43)$ & $0.028^{*}$ \\
\hline Temporal pacemaker, $n(\%)$ & $10(10)$ & $0(0)$ & $10(11)$ & 0.169 \\
\hline Cardioversion, $n(\%)$ & $6(6)$ & $0(0)$ & $6(7)$ & 0.297 \\
\hline ICU hospitalization, $n(\%)$ & $63(61)$ & $5(33)$ & $58(66)$ & $0.017^{*}$ \\
\hline ICU hospitalization $(\text { days })^{\dagger}$ & $2.0(0-5.0)$ & $1.0(0-2.0)$ & $3.0(0-6.5)$ & $0.005^{*}$ \\
\hline Hospitalization (days) ${ }^{\dagger}$ & $14.0(7.0-28.0)$ & $3.0(3.0-9.0)$ & $17.0(8.0-32.0)$ & $0.001^{*}$ \\
\hline In-hospital cardiac mortality, $n(\%)$ & $0(0)$ & $0(0)$ & $0(0)$ & - \\
\hline Cardiac mortality during followup, $n(\%)$ & $0(0)$ & $0(0)$ & $0(0)$ & - \\
\hline Mortality during followup, $n(\%)$ & $14(14)$ & $0(0)$ & $14(16)$ & 0.097 \\
\hline Recurrence, $n(\%)$ & $0(0)$ & $0(0)$ & $0(0)$ & - \\
\hline
\end{tabular}

IABP: intra-aortic balloon pump; ACEI: angiotensin converting enzyme inhibitor; ARB: angiotensin receptor blocker; ICU: intensive care unit.

${ }^{\dagger}$ Presented as median (inter-quartile range).

* Significant finding. 
troponin-I, and NT-proBNP levels. Other group required more frequent hemodynamic support and had significantly longer intensive care unit and in-hospital durations.

In contrast with the previously published data [5-8], our study showed that physical stress, rather than emotional stress, was the predominant triggering event for TTC. TTC cases associated with sepsis and respiratory failure without obstructive coronary artery disease were already reported [6-8], and several hypotheses have been proposed including catecholamine-mediated cardiotoxicity, spasm of the epicardial and/or microvascular coronary circulation, and endothelial cell dysfunction $[15,23,24]$. It was also surprising to know that TTC could occur during or after uneventful elective procedures or surgery performed under regional or general anesthesia.

In the present study, most clinical features of E group were similar to those of other group. The overlapping clinical features in all these presentations may suggest that myocardial stunning resulting from emotional stress may share a common mechanism with those from physical stress, which has been described after subarachnoid hemorrhage and ischemic stroke which is believed to be mediated by catecholamine [15].

Our study showed that E group had significantly higher prevalence of chest pain and palpitation than other group. Some studies reported that catecholamine-mediated myocardial injury has been observed postmortem in people who died under terrifying circumstances such as violent assault, suggesting that catecholamine may be an important link between emotional stress and cardiac injury [15]. Therefore, we supposed that these subjective symptoms predominant in E group might reflect the emotional stressful event might trigger an excessive release of catecholamine, resulting in the presentations of these subjective symptoms and these patients appear to be more vulnerable to sympathetically mediated myocardial stunning or injury.

Interestingly, in our study, other group had significantly higher prevalence of cardiogenic shock, but lower LVEF than E group. Also, other group had significantly higher hs-CRP, peak CK-MB, troponin-I, NT-proBNP, and LVEDP levels than E group. These observations may suggest that patients with idiopathic or physical stressors have more cardiovascular impairment and that perhaps other factors such as significant underlying comorbidities contribute to myocardial stunning or injury. The possible explanations may include myocardial stunning, inflammation, and microvascular spasm in idiopathic/physically stressed TTC patients $[15,16]$. On the other hand, our patients with preceding emotional stressors had relatively preserved myocardial function with higher LVEF and lower LVEDP on initial presentation. These findings may suggest a transient catecholamine-induced myocardial stunning in this group. Therefore, we reasoned that patients with idiopathic or physical stressors had the greater extent of affected myocardium and cardiac markers such as CK-MB and troponin-I may reflect this extent of affected myocardium.

In contrast with recently published study showing that emotional stress group was younger than idiopathic/physical stress group [9], our data reported that the groups did not differ significantly in terms of age between two groups. The possible hypothesis for this discrepancy is that racial differences may affect emotional stressor and susceptibility of TTC with aging. Also, this discrepancy may be related to racial differences in activated sympathetic tones after a stressful event.

A remarkable finding of our study was that sixty-seven $(65 \%)$ of 103 patients had a prolonged corrected QT interval in the acute phases of TTC. Also, E group had significantly higher corrected QT interval, but lower heart rate than other group. These findings were similar to the findings of previous reports showing the associations between TTC and QT prolongation [17-20]. Seth et al. reported 12 cases of TTC with an average corrected QT interval of $478 \mathrm{~ms}$ [18]. Similarly, Abe et al. described 17 patients, most of whom had a prolonged corrected QT interval in the acute and subacute phases of the condition [19]. The QT interval normalized in all cases between 97 and 191 days from the onset of symptoms [19]. It is, therefore, reasonable to regard TTC as a transient acquired insult upon myocardial repolarization that may relate to an acute disturbance of cardiac autonomic function [17-21]. Based on these findings, we supposed that differences of these insults upon myocardial repolarization with triggering stressors may affect different QT intervals between the two groups. Further research is required to study our hypothesis about these differences.

Notably, in our study, there were no differences in the use of inotropic agents, IABP, use of ACEI or ARB, and use of beta blocker between two groups. However, other group had significantly higher prevalence of diuretic use and frequency of ICU stay and had significantly longer durations of in-hospitalization and ICU stay than E group. Moreover, other group required more frequent hemodynamic support and had significantly higher prevalence of diuretic use, and significantly longer duration of hospitalization and ICU stay than $\mathrm{E}$ group. Based on findings of aforementioned studies $[14,22-24]$, in addition to the results of the present study, we reasoned that clinicians should monitor idiopathic/physically stressed TTC patients carefully for hemodynamic compromise. In our study, in-hospital and follow-up cardiac mortalities were $0 \%$, respectively, in TTC patients with idiopathic/physical stressors. These findings may emphasize that the prognosis of TTC itself may be excellent if a meticulous therapeutic strategy under careful monitoring is performed in these patients, particularly in haemodynamic instability.

In the present study, the overall mortality (16\%) was relatively higher than in previous studies [1-4]. However, the overall mortality associated with TTC itself was $0 \%$. It was comparable to results of published reports in other areas of the world [1-4]. During the follow-up period of 5.1 years, most patients (57\%) died of malignancy in our study. According to previously published reports, malignancies may be associated with TTC, potentially as a result of paraneoplastic phenomena $[25,26]$. These findings may indicate that patients with TTC have an excellent prognosis in the absence of significant underlying comorbidities such as malignancy or stroke. 


\section{Study Limitations}

There are some limitations that should be considered in our study. First, this was a retrospective analysis. Second, the results of our study may be limited by the relatively small number of patients. Third, we did not perform systemic investigations such as catecholamine measurements, magnetic resonance imaging, viral antibody titers, or pathology. Because TTC is a kind of exclusion diagnosis, some patients with other diseases, such as myocarditis, may be misdiagnosed in our study. However, diagnosis of TTC on the basis of clinical presentation and characteristic wall motion abnormality is realistic and often used in many studies.

\section{Conclusions}

The clinical features of TTC are different between groups with and without preceding emotional stressors. The TTC group with preceding emotional stressors was more likely to have preserved cardiovascular reserve and lesser likely to require hemodynamic support than other group despite the entire prognosis of TTC is excellent regardless of triggering stressors.

\section{Conflict of Interests}

The authors report no financial relationships or conflict of interests regarding the content herein.

\section{References}

[1] S. Kurisu, H. Sato, T. Kawagoe et al., "Tako-tsubo - like left ventricular dysfunction with ST-segment elevation: a novel cardiac syndrome mimicking acute myocardial infarction," American Heart Journal, vol. 143, no. 3, pp. 448-455, 2002.

[2] M. Gianni, F. Dentali, A. M. Grandi, G. Sumner, R. Hiralal, and E. Lonn, "Apical ballooning syndrome or takotsubo cardiomyopathy: a systematic review," European Heart Journal, vol. 27, no. 13, pp. 1523-1529, 2006.

[3] T. M. Pilgrim and T. R. Wyss, "Takotsubo cardiomyopathy or transient left ventricular apical ballooning syndrome: a systematic review," International Journal of Cardiology, vol. 124, no. 3, pp. 283-292, 2008.

[4] A. Prasad, A. Lerman, and C. S. Rihal, "Apical ballooning syndrome (Tako-Tsubo or stress cardiomyopathy): a mimic of acute myocardial infarction," American Heart Journal, vol. 155, no. 3, pp. 408-417, 2008.

[5] S. W. Sharkey, D. C. Windenburg, J. R. Lesser et al., "Natural history and expansive clinical profile of stress (Tako-Tsubo) cardiomyopathy," Journal of the American College of Cardiology, vol. 55, no. 4, pp. 333-341, 2010.

[6] Y. P. Lee, K. K. Poh, C. H. Lee et al., "Diverse clinical spectrum of stress-induced cardiomyopathy," International Journal of Cardiology, vol. 133, no. 2, pp. 272-275, 2009.

[7] N. K. Singh, S. Rumman, F. L. Mikell, N. Nallamothu, and C. Rangaswamy, "Stress cardiomyopathy: clinical and ventriculographic characteristics in 107 North American subjects," International Journal of Cardiology, vol. 141, no. 3, pp. 297303, 2010.

[8] V. Vidi, V. Rajesh, P. P. Singh et al., "Clinical characteristics of Tako-Tsubo cardiomyopathy," American Journal of Cardiology, vol. 104, no. 4, pp. 578-582, 2009.
[9] Y. Yaqub, L. A. Jenkins, J. Suarez et al., "Emotional stress and Tako-Tsubo cardiomyopathy: observations on 2 distinct clinical phenotypes," Journal of Investigative Medicine, vol. 58, no. 2, pp. 298-302, 2010.

[10] B. G. Song, J. Y. Hahn, S. J. Cho et al., "Clinical characteristics, ballooning pattern, and long-term prognosis of transient left ventricular ballooning syndrome," Heart and Lung, vol. 39, no. 3, pp. 188-195, 2010.

[11] V. Menon, H. White, T. LeJemtel, J. G. Webb, L. A. Sleeper, and J. S. Hochman, "The clinical profile of patients with suspected cardiogenic shock due to predominant left ventricular failure: a report from the SHOCK Trial Registry," Journal of the American College of Cardiology, vol. 36, no. 3, pp. 1071-1076, 2000.

[12] R. M. Lang, M. Bierig, R. B. Devereux et al., "Recommendations for chamber quantification: a report from the American Society of Echocardiography's guidelines and standards committee and the Chamber Quantification Writing Group, developed in conjunction with the European Association of Echocardiography, a branch of the European Society of Cardiology," Journal of the American Society of Echocardiography, vol. 18, no. 12, pp. 1440-1463, 2005.

[13] W. A. Zoghbi, M. Enriquez-Sarano, E. Foster et al., "Recommendations for evaluation of the severity of native valvular regurgitation with two-dimensional and doppler echocardiography," Journal of the American Society of Echocardiography, vol. 16, no. 7, pp. 777-802, 2003.

[14] B. G. Song, S. J. Park, H. J. Noh et al., "Clinical characteristics, and laboratory and echocardiographic findings in takotsubo cardiomyopathy presenting as cardiogenic shock," Journal of Critical Care, vol. 25, no. 2, pp. 329-335, 2010.

[15] I. S. Wittstein, D. R. Thiemann, J. A. C. Lima et al., "Neurohumoral features of myocardial stunning due to sudden emotional stress," New England Journal of Medicine, vol. 352, no. 6, pp. 539-548, 2005.

[16] K. Sadamatsu, H. Tashiro, N. Maehira, and K. Yamamoto, "Coronary microvascular abnormality in the reversible systolic dysfunction observed after noncardiac disease," Japanese Circulation Journal, vol. 64, no. 10, pp. 789-792, 2000.

[17] E. R. Behr and S. Mahida, "Takotsubo cardiomyopathy and the long-QT syndrome: an insult to repolarization reserve," Europace, vol. 11, no. 6, pp. 697-700, 2009.

[18] P. S. Seth, G. P. Aurigemma, J. M. Krasnow, D. A. Tighe, W. J. Untereker, and T. E. Meyer, "A syndrome of transient left ventricular apical wall motion abnormality in the absence of coronary disease: a perspective from the United States," Cardiology, vol. 100, no. 2, pp. 61-66, 2003.

[19] Y. Abe, M. Kondo, R. Matsuoka, M. Araki, K. Dohyama, and H. Tanio, "Assessment of clinical features in transient left ventricular apical ballooning," Journal of the American College of Cardiology, vol. 41, no. 5, pp. 737-742, 2003.

[20] H. Furushima, M. Chinushi, A. Sanada, and Y. Aizawa, "Ventricular repolarization gradients in a patient with takotsubo cardiomyopathy," Europace, vol. 10, no. 9, pp. 1112-1115, 2008.

[21] Y. J. Akashi, G. Barbaro, T. Sakurai, K. Nakazawa, and F. Miyake, "Cardiac autonomic imbalance in patients with reversible ventricular dysfunction takotsubo cardiomyopathy," QJM, vol. 100, no. 6, pp. 335-343, 2007.

[22] G. Cocco and D. Chu, "Stress-induced cardiomyopathy: a review," European Journal of Internal Medicine, vol. 18, no. 5, pp. 369-379, 2007. 
[23] J. Lindsay, A. Paixao, T. Chao, and A. D. Pichard, "Pathogenesis of the takotsubo syndrome: a unifying hypothesis," American Journal of Cardiology, vol. 106, no. 9, pp. 1360-1363, 2010.

[24] I. Pernicova, S. Garg, C. V. Bourantas, F. Alamgir, and A. Hoye, "Takotsubo cardiomyopathy: a review of the literature," Angiology, vol. 61, no. 2, pp. 166-173, 2010.

[25] C. Burgdorf, H. M. Nef, D. Haghi, V. Kurowski, and P. W. Radke, "Tako-tsubo (stress-induced) cardiomyopathy and cancer," Annals of Internal Medicine, vol. 152, no. 12, pp. 830$831,2010$.

[26] C. Burgdorf, V. Kurowski, H. Bonnemeier, H. Schunkert, and P. W. Radke, "Long-term prognosis of the transient left ventricular dysfunction syndrome (Tako-Tsubo cardiomyopathy): focus on malignancies," European Journal of Heart Failure, vol. 10, no. 10, pp. 1015-1019, 2008. 


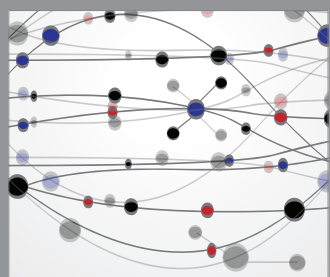

The Scientific World Journal
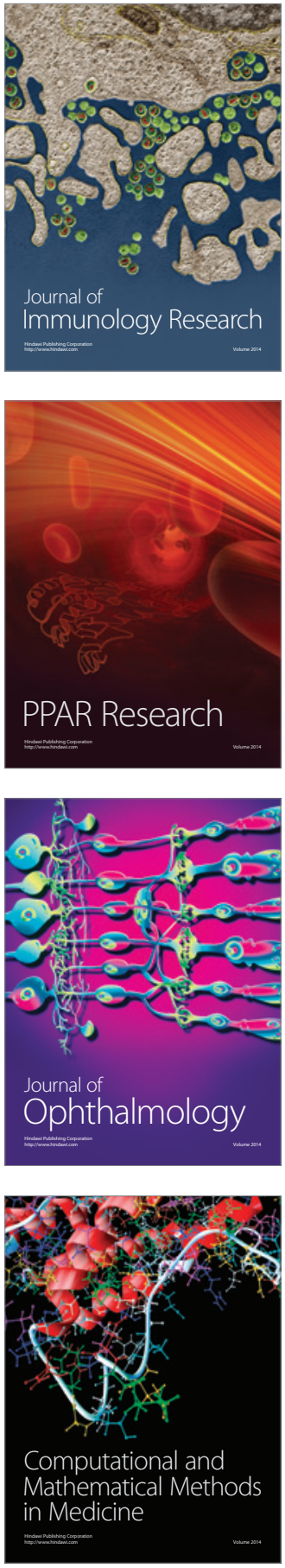

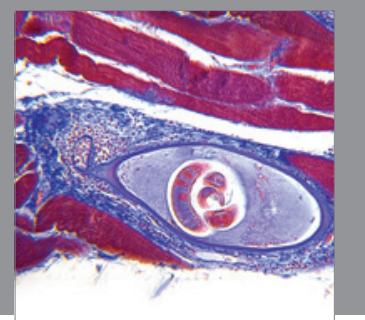

Gastroenterology

Research and Practice
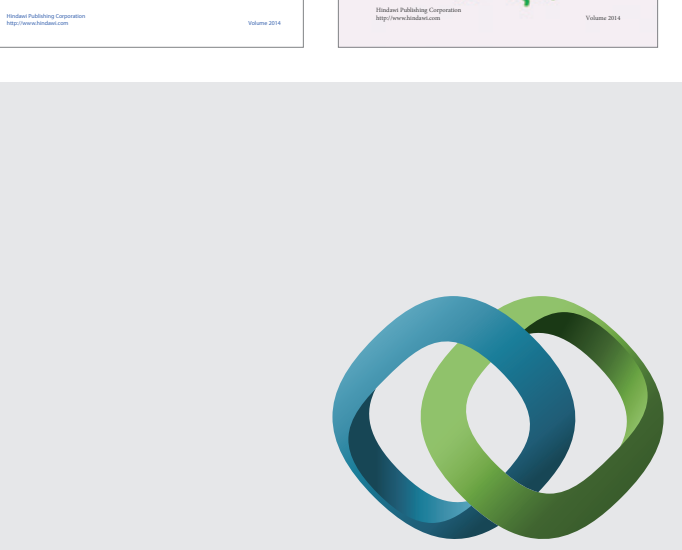

\section{Hindawi}

Submit your manuscripts at

http://www.hindawi.com
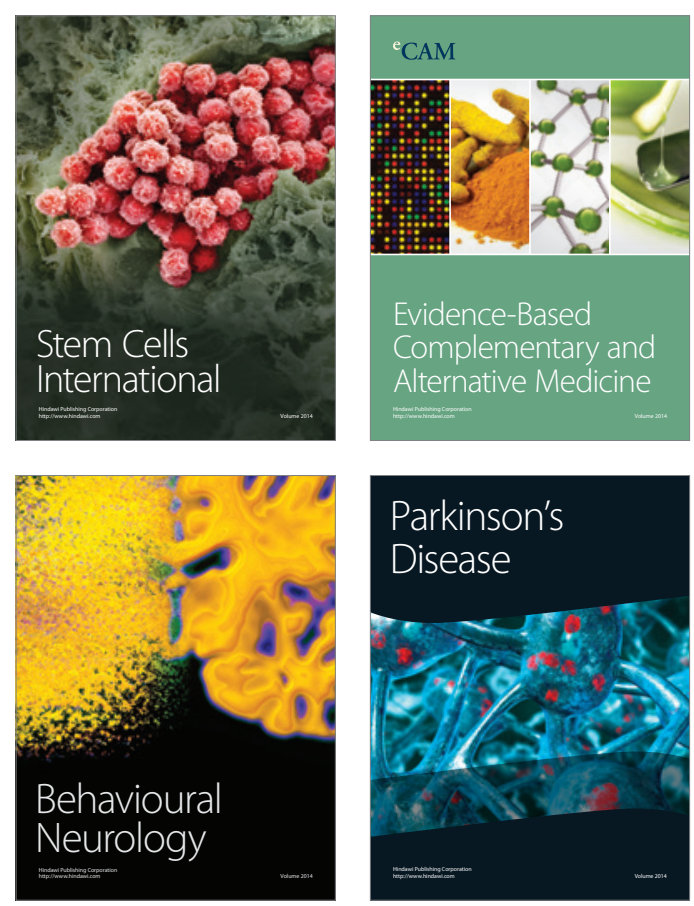

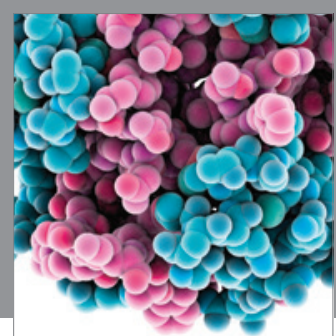

Journal of
Diabetes Research

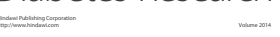

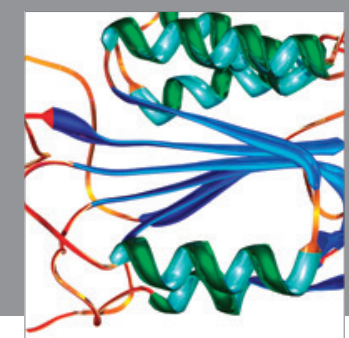

Disease Markers
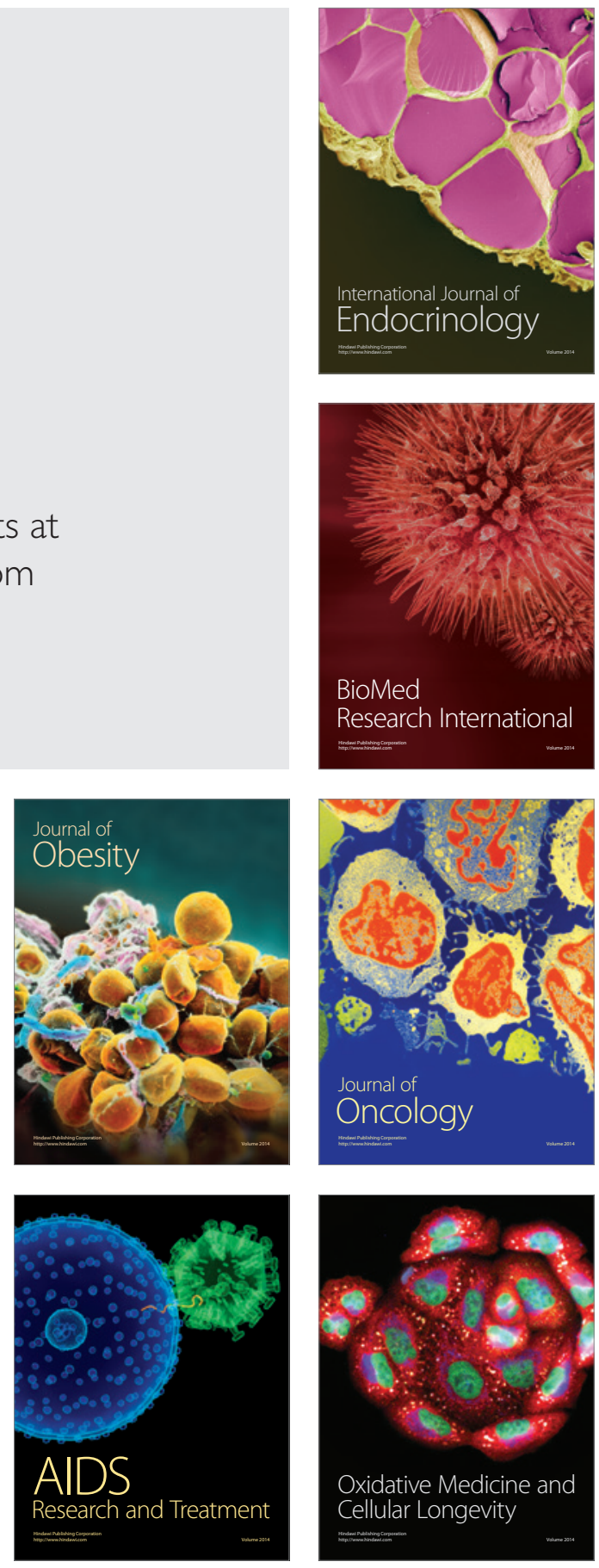